\title{
A novel injectable calcium phosphate-based nanocomposite for the augmentation of cannulated pedicle-screw fixation
}

This article was published in the following Dove Press journal:

International Journal of Nanomedicine

27 April 2017

Number of times this article has been viewed

\author{
Haolin Sun ${ }^{1, *}$ \\ Chun $\mathrm{Liu}^{2, *}$ \\ Huiling $\mathrm{Liu}^{2}, *$ \\ Yanjie $\mathrm{Bai}^{3}$ \\ Zheng Zhang' \\ Xuwen $\mathrm{Li}^{\prime}$ \\ Chunde $\mathrm{Li}^{1}$ \\ Huilin Yang 2,4 \\ Lei Yang ${ }^{2,4}$ \\ 'Department of Orthopedics, Peking \\ University First Hospital, Beijing, \\ ${ }^{2}$ Department of Orthopedics, Institute \\ of Orthopedics, First Affiliated \\ Hospital, ${ }^{3}$ School of Public Health, \\ Medical College, ${ }^{4}$ International \\ Research Center for Translational \\ Orthopedics (IRCTO), Soochow \\ University, Suzhou, China \\ *These authors contributed equally \\ to this work
}

\begin{abstract}
Polymethyl methacrylate (PMMA)-augmented cannulated pedicle-screw fixation has been routinely performed for the surgical treatment of lumbar degenerative diseases. Despite its satisfactory clinical outcomes and prevalence, problems and complications associated with high-strength, stiff, and nondegradable PMMA have largely hindered the long-term efficacy and safety of pedicle-screw fixation in osteoporotic patients. To meet the unmet need for better bone cement for cannulated pedicle-screw fixation, a new injectable and biodegradable nanocomposite that was the first of its kind was designed and developed in the present study. The calcium phosphate-based nanocomposite (CPN) exhibited better anti-pullout ability and similar fluidity and dispersing ability compared to clinically used PMMA, and outperformed conventional calcium phosphate cement (CPC) in all types of mechanical properties, injectability, and biodegradability. In term of axial pullout strength, the $\mathrm{CPN}$-augmented cannulated screw reached the highest force of $\sim 120 \mathrm{~N}$, which was higher than that of PMMA $(\sim 100 \mathrm{~N})$ and CPC $(\sim 95 \mathrm{~N})$. The compressive strength of the CPN (50 MPa) was three times that of $\mathrm{CPC}$, and the injectability of the CPN reached $95 \%$. In vivo tests on rat femur revealed explicit biodegradation of the $\mathrm{CPN}$ and subsequent bone ingrowth after 8 weeks. The promising results for the CPN clearly suggest its potential for replacing PMMA in the application of cannulated pedicle-screw fixation and its worth of further study and development for clinical uses.
\end{abstract}

Keywords: calcium phosphate nanocomposite, injectable, biodegradable, pedicle screw, lumbar degenerative disease, osteoporosis

\section{Introduction}

Due to great advantages in ensuring the effect of decompression, stabilizing spine structure, and promoting fusion-rate and rehabilitation results at an early stage, lumbar pedicle-screw fixation and fusion have become the gold standard for the treatment of lumbar degenerative diseases, such as degenerative lumbar stenosis, degenerative lumbar scoliosis, and lumbar spondylolisthesis. However, pedicle-screw fixation in severely osteoporotic spine remains a challenge for orthopedic surgeons, and its efficacy in osteoporotic patients is still questionable. The reduction of bone mass in osteoporotic patients greatly decreases the fixation strength of pedicle screws, causing loosening or pullout of screws, cutout of the vertebral body, failure of fixation, and a number of potential risks or complications. ${ }^{1-3}$

Augmentation with bone cement has been demonstrated to be an effective way to increase the fixation strength of pedicle screws in osteoporotic patients. Previous studies have reported that the pullout strength of pedicle screws can be dramatically increased by augmentation with nondegradable or bioabsorbable bone cements such 
as polymethyl methacrylate (PMMA), hydroxyapatite (HA), calcium phosphates, or calcium sulfates. ${ }^{4-10}$ Among these bone cements, PMMA is the only clinically approved cement for the augmentation of pedicle-screw fixation. In the routine surgery of augmentation using bone cement, a pilot hole is drilled first and filled with PMMA prior to inserting the screw. This surgical route raises problems, such as high risk of PMMA leakage, low repeatability due to the complex procedure, and failure of screw insertion. ${ }^{9}$ In order to solve these problems, cannulated and fenestrated pedicle screws have been invented. During surgery, PMMA bone cement is injected into the cannula and flows out from the side holes of the screw, infiltrating into cancellous bone of vertebra and forming a screw-cement-bone complex. This complex can promote the mechanical stability of screw fixation, improve fatigue resistance, and also decrease the risk of cement leakage. ${ }^{11-13}$ To date, all the clinical cases and most of the research have used only PMMA for the augmentation of cannulated screws, ${ }^{14,15}$ largely due to the high fluidity and injectability of PMMA, which can easily be fed through a cannula of a typical diameter of $1.6 \mathrm{~mm}$ and outlet holes of small diameter - around $0.9 \mathrm{~mm}$. Other types of bioabsorbable cements are comparatively less reported for applications in cannulated pedicle screws.

The clinical efficacy of PMMA-augmented pedicle-screw fixation has been demonstrated in the treatment of lumbar degenerative diseases, yet problems and controversy have also emerged amid the complications and problems associated with PMMA. PMMA is a stiff and high-strength epoxy that has large discrepancy in mechanical properties compared to bone, especially osteoporotic bone. This biomechanical mismatch creates shielding effects on adjacent vertebral bodies, eventually causing compressive fracture or adjacent disk disease. ${ }^{16}$ Also, in cases where pedicle screws need to be retrieved or removed from the screw-cement-bone complex, this becomes extremely difficult or even impossible, due to the excessive bonding strength between screws and the cement. ${ }^{17}$ Most importantly, due to its nondegradability and inertness, PMMA cannot form a biological bonding with bone, nor can it induce bone ingrowth or integration. In addition, when considering the cases of postoperation infection, the nondegradable PMMA becomes another risk factor for both deep infection and superficial infection. ${ }^{16}$

These drawbacks associated with PMMA yield an unmet need of robust but bioactive or bioabsorbable bone cement for better biomechanical outcomes of cannulated pediclescrew fixation. In the present study, a novel injectable calcium phosphate-based nanocomposite (CPN) was designed and prepared to meet the needs of cannulated pedicle-screw fixation for improved efficacy and biomechanical stability. Our previous work has reported the effect of a starch network on improving the mechanical strengths of calcium phosphate cement (CPC). ${ }^{18,19}$ The hypothesis here is that this CPN may possess similar biomechanical properties to the clinically used PMMA for application in cannulated pedicle-screw augmentation. Also, its superior biodegradability to PMMA may lower the risk of screw-associated complications, such as infection and difficulty in removing screws. The rationale in this work was modifying self-setting, bioabsorbable CPCs with a nanoscale starch network, forming a reinforced but injectable nanocomposite that augments cannulated screw fixation and induces osseointegration. This study focused on the biomechanical properties of the CPN in the augmentation of cannulated screw fixation in comparison with traditional solid screws and PMMA-augmented cannulated screws. It also demonstrated the degradability and osseointegrative ability of the CPN. This study is expected to provide insights and important reference for further clinical applications of CPN.

\section{Materials and methods Preparation of CPC and CPN}

Analytical dicalcium phosphate dihydrate (DCPD; SigmaAldrich, St Louis, MO, USA), sodium hydrogen phosphate $\left(\mathrm{Na}_{2} \mathrm{HPO}_{4}\right.$, Sigma-Aldrich), barium sulfate $\left(\mathrm{BaSO}_{4}(\mathrm{BS})\right.$, Sigma Aldrich), and $\alpha$-tricalcium phosphate ( $\alpha$-TCP; Dingan Science and Technology, Suzhou, China) were ball-milled in ethanol before use. Base materials of CPC were prepared followed the formula reported in our previous study, which contained $\alpha$-TCP and DCPD at a mass ratio of 9:1. ${ }^{18} \mathrm{Com}-$ mercially available, cost-efficient starch (Jingrui New Material, Xuancheng, China) underwent a gelatinization process when added in water to form a nanoscale network consisting of starch molecular chains, which was confirmed by transmission electron microscopy (TEM; HT7700, 200 kV; Hitachi, Tokyo, Japan). The solid content of the CPN was composed of CPC ( $80 \mathrm{wt} \%)$ and a mixture of starch and BS, and the setting liquid was $0.25 \mathrm{~mol} / \mathrm{L} \mathrm{Na}_{2} \mathrm{HPO}_{4}$ solution. For comparison purposes, $\mathrm{CPC}$ with $20 \mathrm{wt} \% \mathrm{BS}$ (CPC-BS) was also prepared using a similar method.

To prepare the cement paste for injection through cannulated pedicle screws, the solid content of CPC, CPC-BS, or CPN was mixed with the setting liquid at a liquid:solid (L:S) ratio of $0.8-1.4 \mathrm{~mL} / \mathrm{g}$, depending on injection requirements. The cement paste was injected through a syringe connected to the cannula of the pedicle screw or directly to the osteoporotic bone model or mold for biomechanical evaluations. 


\section{Characterization of CPC and CPN}

The microstructure of the CPC or CPN was directly observed by scanning electron microscopy (SEM; Quanta 250; Thermo Fisher Scientific, Waltham, MA, USA) on the fracture surfaces of hardened cement bars (at $37^{\circ} \mathrm{C}$ for at least 24 hours). The surfaces were precoated with an Au-Pd layer before SEM observation. Phase compositions of CPC and CPN specimens hardened for 3 days and the $\mathrm{CPC}$ before reaction were examined by wide-angle X-ray diffraction (XRD; X'Pert Pro MRD; PANalytical, Almelo, Netherlands) using $\mathrm{CuK} \alpha$ radiation $(\lambda=1.5402 \AA)$. The $2 \theta$ diffraction angle was $10^{\circ}-60^{\circ}$ in a step-scan mode of $2^{\circ}$ per minute. In addition, original CPC powder before reaction, CPC, CPC-BS, and CPN powder after setting for 3 days were ground into powder and analyzed by Fourier-transform infrared spectroscopy (FTIR; Nicolet 6700; Thermo Fisher Scientific) in the $400-4,000 \mathrm{~cm}^{-1}$ range.

\section{Pedicle screws, PMMA cement, and osteoporotic bone model}

Both traditional and cannulated pedicle screws (UPass and MISpine, respectively; Weigao Orthopaedic Device, Weihai, China) were made of Ti6Al4V and had outer diameter of $6.5 \mathrm{~mm}$, length of $45 \mathrm{~mm}$, and cannula diameter of $1.6 \mathrm{~mm}$. The screws were received in sterile packages and thus directly used for study. For comparison purposes, clinically used PMMA cement (Mendec Spine; Tecres SPA, Sommacampagna, Italy) was used following the manufacturer's instructions.

For the in vitro osteoporotic bone model, commercially available synthetic composite bone was used (Sawbones model 1522-507; Pacific Research Laboratories, Vashon, WA, USA), with a density of $0.09 \mathrm{~g} / \mathrm{cm}^{3}$ and $95 \%$ opencelled structure. The Sawbones mimicked human vertebrae with severe osteoporosis and provided a homogeneous and consistent material similar to human cancellous bone, as well as X-ray inspection capacity. ${ }^{20}$

\section{Degradability and injectability}

The degradability of the CPN was investigated in vitro by immersing the hardened cylindrical CPN samples in Tris$\mathrm{HCl}$ solution at $37^{\circ} \mathrm{C}$ using an S:L ratio of $0.2 \mathrm{~g} / \mathrm{mL}$. The cylindrical samples were prepared by injecting the $\mathrm{CPN}$ paste into a cylindrical stainless-steel mold of $6 \mathrm{~mm}$ diameter and $12 \mathrm{~mm}$ length and setting for 2 minutes. The sample was pushed out from the mold and further hardened at $37^{\circ} \mathrm{C}$ and $100 \%$ relative humidity for 3 days. The weight change of $\mathrm{CPN}$ cylinders immersed in Tris- $\mathrm{HCl}$ solution was measured every 7 days, and the percentage of weight loss with respect to original weight was calculated.

The injectability of the CPN and PMMA was tested by a standardized method reported elsewhere. ${ }^{19}$ Briefly, when the cement started to set, the paste was placed into a $5 \mathrm{~mL}$ syringe with a needle of $1.6 \mathrm{~mm}$ inner diameter and extruded under a force less than $50 \mathrm{~N}$. The weight percentage of the extruded paste with respect to the total paste was defined as injectability. The test for each cement formulation was repeated three times.

\section{Fluidity and dispersion ability}

Fluidity and dispersion of cements in osteoporotic bone were evaluated by injecting the pastes into $4 \times 4 \times 2 \mathrm{~cm}^{3}$ Sawbones blocks and measuring the geometry and volume of the dispersed and hardened pastes. The L:S ratio of PMMA cement was 0.45 , as suggested by the manufacturer, while CPC and the CPN were tested for different $\mathrm{L}: \mathrm{S}$ ratios of $0.8,1,1.2$, 1.4, and 1.6. The paste was hardened within the Sawbones block at body temperature for 1 day, and the cement lump was extracted for evaluation. Then, orthogonal projection areas of cement lumps were measured. To assess the volume of the cement lumps, the orthogonal projection areas were equalized to two elliptical areas, from which a ellipsoidal volume was calculated. Large projection area or volume of the lumps was indicative of high fluidity and dispersion in the Sawbones.

\section{Biomechanical properties}

Basic mechanical properties of bone cements

Basic mechanical properties of bone cements were evaluated by uniaxial compression tests, according to methods previously reported. ${ }^{18,19}$ The cement was set in a cylindrical stainless-steel mold ( $6 \mathrm{~mm}$ in diameter and $12 \mathrm{~mm}$ in length), and the end planes of cement bars were smoothed with 800 -grade sandpaper. The cement bars were then uniaxially compressed by a mechanical tester (HY-1080; Heng Yi Precision Instrument, Shanghai, China) with a $10 \mathrm{kN}$ load cell (gauge precision $0.5 \mathrm{~N}$ ) at a crosshead speed of $0.5 \mathrm{~mm} /$ min. Stress-strain curves were collected. Maximum stress was taken as compressive strength and the linear range in the stress curve before failure was used to calculate the compressive modulus. Each type of cement was tested at least five times.

\section{Implementation of screw and bone cement}

Both traditional (solid) and cannulated pedicle screws were implemented with different types of cements in the 
$5 \times 4 \times 2 \mathrm{~cm}^{3}$ Sawbones blocks. In the case of solid screws, a guiding screw of smaller diameter $(6 \mathrm{~mm})$ was prescrewed into the Sawbones block to create a canal and $1.5 \mathrm{~mL}$ PMMA, CPC (with or without BS), or CPN cement was injected into the bottom of the canal and the solid screw was then screwed in. In the case of cannulated screw, it was screwed into the Sawbones block, and $1.5 \mathrm{~mL}$ bone cement was injected through the cannula. Blocks with both screw and cement were placed in an oven at $37^{\circ} \mathrm{C}$ for 24 hours before mechanical tests. The L:S ratio used for $\mathrm{CPC}$ and the $\mathrm{CPN}$ was $0.8 \mathrm{~mL} / \mathrm{g}$. Before further tests, X-ray radiographs of bone cements combined with different types of screws within Sawbones blocks were collected using a veterinary X-ray machine (radiation $60 \mathrm{kV}$ and $2.5 \mathrm{mAs}$; SPL-HF-VET-4.0, SEDECAL, Spain).

\section{Axial pullout strength}

Each Sawbones block containing the screw and cement was placed in two clamped L-shaped metal holders and the head of screw clamped vertically to a universal mechanical testing machine (HY-1080; Hengyi Group, Shanghai, China). The Sawbones block remained static while the screw was pulled out at a speed of $2 \mathrm{~mm} / \mathrm{min}$ using an axial tensile testing mode. The load-displacement curve was measured till the screw-cement complex was completely pulled out from the Sawbones block. Photographs of the screw-cement complex were also taken. The maximum pullout force before failure was determined as the axial pullout strength. Each type of cement was tested at least five times.

\section{Maximum torque}

The sample setup for torsion tests was similar to that of the pullout test. Instead of pullout, the screw was rotated clockwise at a constant rate of $0.5^{\circ}$ second along the direction of the screw axis. The prestress for torsion test was $10 \mathrm{~N}$. The torque was applied until the torque value decreased significantly when the screw or cement detached from the Sawbones block, and a torque-angle curve was recorded. Maximum torque was read from the curve, and each type of cement was tested at least five times.

\section{In vivo osseointegration of CPN}

The bone-defect model on two Sprague Dawley rats weighing 200-250 g was used to evaluate the osseointegration capability of the CPN in vivo. The animal-study protocol was approved by the ethics committee of Soochow University (2015-8152200133) and followed the Guide for the Care and Use of Laboratory Animals (eighth edition). The rats were anesthetized using an intramuscular injection of $100 \mathrm{mg} / \mathrm{kg}$ ketamine hydrochloride and $100 \mathrm{mg} / \mathrm{kg}$ xylazine. After hind limbs had been shaved and sterilized, a longitudinal incision was made to expose the metaphyseal region of the distal femur. A bone defect of $2.5 \mathrm{~mm}$ diameter and $3 \mathrm{~mm}$ depth was created, and the defect was filled with a CPN cylinder prepared as described earlier. After 8 weeks, the rats were killed and histological analysis on the defect region performed. Thin sections $(5 \mu \mathrm{m})$ were sliced perpendicularly through the implants using a soft-tissue slicer. The slice was stained with hematoxylin and eosin (H\&E) staining and examined by optical microscopy.

\section{Statistics}

Data were reported as mean \pm standard deviation calculated from repeated tests. Statistical analysis was conducted by one-way analysis of variance. All pairwise comparisons were performed with Tukey's post hoc test.

\section{Results}

\section{Characterization of CPC and CPN}

TEM image (Figure 1A) shows the nanoscale networks of gelatinized starch, which were further incorporated with CPC and BS to form the CPN. Gelatinized starch
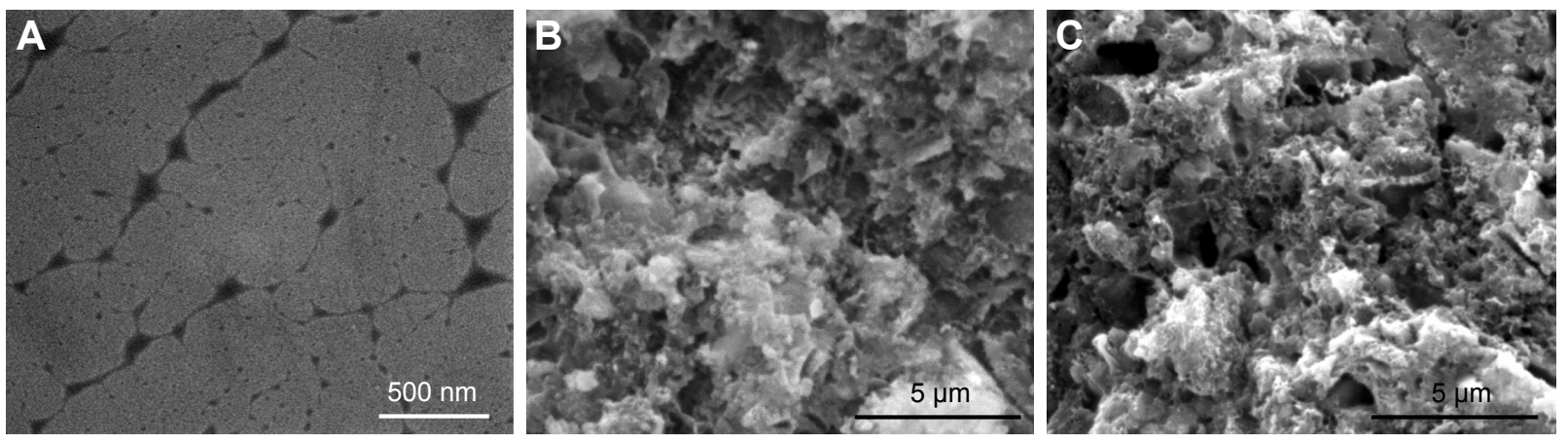

Figure I (A) TEM image of nanoscale networks of gelatinized starch; SEM image of microscopic morphology of fractured surfaces of (B) CPC and (C) CPN. Abbreviations: TEM, transmission electron microscopy; SEM, scanning electron microscopy; CPC, calcium phosphate cement; CPN, calcium phosphate nanocomposite. 
Table I Compressive strengths and moduli of bone cements

\begin{tabular}{lll}
\hline Cement & Strength (MPa) & Modulus (GPa) \\
\hline CPC & $13.21 \pm 2.45$ & $0.47 \pm 0.15$ \\
CPC-BS & $17.02 \pm 2.66$ & $1.54 \pm 0.19$ \\
CPN & $50.17 \pm 3.8$ & $2.5 \pm 0.25$ \\
PMMA & $92.18 \pm 10.91$ & $1.31 \pm 0.11$ \\
\hline
\end{tabular}

Note: Data presented as mean \pm standard deviation.

Abbreviations: $\mathrm{CPC}$, calcium phosphate cement; $\mathrm{BS}, \mathrm{BaSO}_{4} ; \mathrm{CPN}$, calcium phosphate nanocomposite; PMMA, polymethyl methacrylate.

formed a viscous gel of disentangled amylose and amylopectin as a result of disintegrations of starch granules by hydration, and the chain-like molecules rearranged into an open network of nanofibers with thickness from tens of nanometers to a few hundred nanometers (as shown in Figure 1A). The nanoscale network of starch was readily mixed with CPC (Figure 1B) and BS powders to form the CPN (Figure 1C), which possessed significantly higher mechanical strength compared to CPC itself (Table 1). There were no microfractures or microcracks after the $\mathrm{CPN}$ had solidified for 3 days. Surface morphology of CPC and the CPN revealed homogeneous mixing of micron-sized ceramic particles and existence of submicron pores, which is typical for CPC-based cement.

The significantly increased mechanical properties of the CPN were demonstrated by much higher compressive strength and modulus compared to CPC and CPC-BS, as shown in Figure 2 and Table 1. The compressive strength of the CPN was almost three times that of CPC and CPC-BS, clearly suggesting the reinforcing effect of the starch

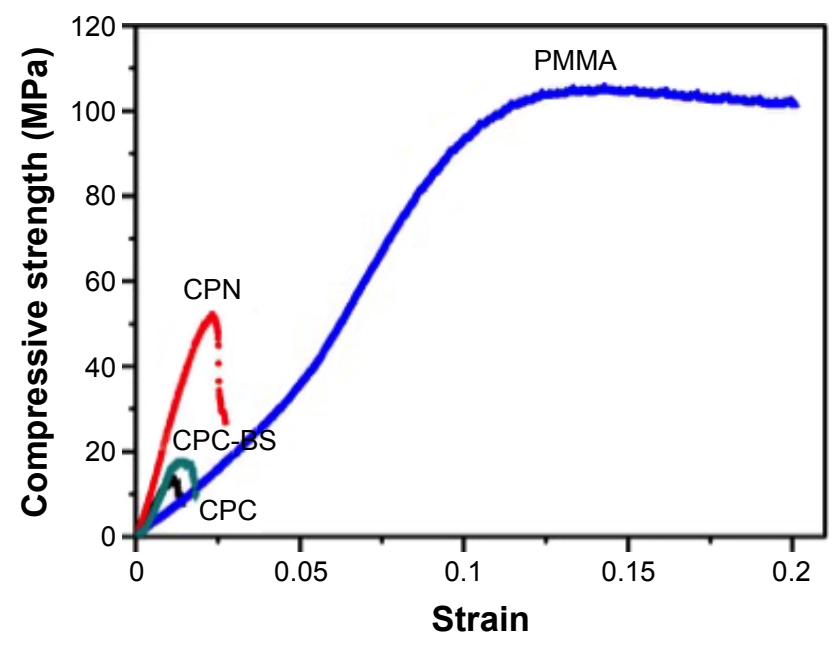

Figure 2 Representative compressive stress-strain curves of PMMA, CPC, CPC$\mathrm{BS}$, and CPN.

Abbreviations: PMMA, polymethyl methacrylate; CPC, calcium phosphate cement: $\mathrm{BS}, \mathrm{BaSO}_{4}$; $\mathrm{CPN}$, calcium phosphate nanocomposite. nanonetwork on the mechanical strength of bone cement. This compressive strength of the CPN was also much higher than natural cancellous bone in the vertebral body, indicating that the CPN had proper mechanical strength for the augmentation of osteoporotic spine. Although the compressive strength of the CPN was only half that of PMMA (50 MPa vs $92 \mathrm{MPa}$ ), the compressive modulus of CPN (2.5 GPa) was almost twice that of PMMA (1.31 GPa). A much stiffer CPN indicated higher capability of resisting compression than softer PMMA at small strains $(<2.5 \%)$, which possibly contributed to the high pullout strengths reported later.

Chemical composition of CPN and CPCs were analyzed by FTIR and XRD (Figure 3). Most of the bands in the FTIR spectra of the cements were attributed to HA crystallized from the reaction of DCPD and $\alpha$-TCP, showing $\mathrm{PO}_{4}{ }^{3-}$-derived bands at 563,606 , and $1,030-1,090 \mathrm{~cm}^{-1}$. The broad bands at 3,140-3,600 $\mathrm{cm}^{-1}$ belong to the -OH in starch and HA, while the 1,030 and $1,120 \mathrm{~cm}^{-1}$ bands may derive from the $\mathrm{C}-\mathrm{O}-\mathrm{C}$ group of starch and $\mathrm{PO}_{4}^{3-}$, respectively. The difference in the spectra of CPC-BS and CPN could be attributed to the gelatinized starch in the cement.

XRD results further confirmed the phase composition of cement samples before and after the hydration reaction of calcium phosphates (Figure 3B). The original CPC system designed in this study completely converted to crystalline HA after 3 days, while radiopaque BS remained and did not affect the reaction of cement. Major inorganic phases of the CPN were $\mathrm{CPC}$ and BS, while the starch nanonetwork appeared to have no impact on the crystallization of HA.

\section{In vitro degradability and injectability}

Unlike the nondegradable PMMA, calcium phosphate-based cements have different biodegradability depending on their composition. The degradation process, evaluated by soaking $\mathrm{CPC}$ and $\mathrm{CPN}$ cements in Tris- $\mathrm{HCl}$ and expressed by the weight-loss percentage of the cements, is shown in Table 2 . Degradation of CPC and the CPN was at the highest rate in the first week of the test, both revealing a $6 \%$ weight loss after 7 days. Degradation slowed down for both cements after 1 week, and a difference in degradability emerged. The CPN showed significantly faster degradation than CPC after the first week, reaching $10.62 \%$ weight loss at the fifth week, while CPC only had a weight loss of $8.7 \%$. This result indicated that the CPN had higher degradability than CPC, which is desirable for vertebral augmentation application, probably due to the disassociation of the nanoscale network of starch in the CPN. 
A

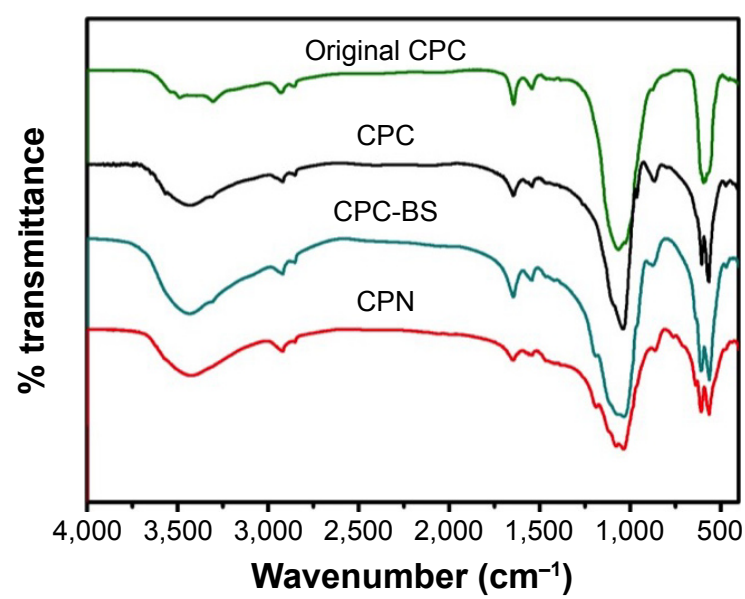

B

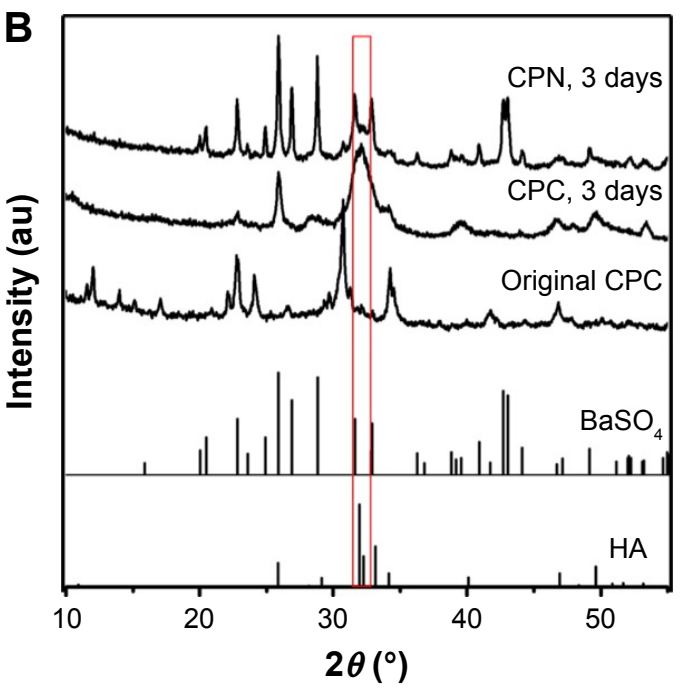

Figure 3 (A) FTIR spectra and (B) XRD patterns of different cements.

Notes: Original CPC was the mixture of powders before solid-liquid reaction and other samples were after setting for 3 days. Standard $\mathrm{XRD}$ spectra of $\mathrm{HA}$ and $\mathrm{BaSO} \mathrm{O}_{4}$ are also shown in (B).

Abbreviations: $\mathrm{CPC}$, calcium phosphate cement; $\mathrm{BS}, \mathrm{BaSO}_{4}$; $\mathrm{CPN}$, calcium phosphate nanocomposite; HA, hydroxyapatite; FTIR, Fourier-transform infrared spectroscopy; $\mathrm{XRD}, \mathrm{X}$-ray diffraction.

The addition of the gelatinized starch nanonetwork to CPC also greatly improved its injectability. CPC paste was prone to liquid-solid separation during the injection, and measured injectability was only $15 \pm 1 \%$. In contrast, CPN paste was ductile, squeezable, and could be injected easily through a canal $1.5 \mathrm{~mm}$ in diameter, and measured injectability reached $95 \pm 1 \%$ when the L:S ratio of the CPN was $0.6 \mathrm{~mL} / \mathrm{g}$. This high injectability of the CPN can meet the requirement for being used in cannulated pedicle screws, and indeed all the CPN was readily injected through a cannula of $1.6 \mathrm{~mm}$ in diameter in the screws.

\section{Fluidity and dispersion ability}

Fluidity and dispersion ability of different bone cements were evaluated by examining the dispersed cement lumps in Sawbones blocks that simulated the osteoporotic spine, and the results are shown in Figure 4. Morphology of the dispersed clumps is shown in Figure 4A, and a spheroid geometry suggested acceptable fluidity of cements for penetration and interdigitation into a sponge-like bone structure. CPC and CPN clumps became ellipsoid when the L:S ratio was over $1.4 \mathrm{~mL} / \mathrm{g}$, suggesting that the fluidity was so high that cement tended to flow away in the sponge bone before hardening.
Quantified results of the projection area and estimated volume of the clumps further confirmed this observation. CPC generally had worst dispersion ability than PMMA and CPN, while the $\mathrm{CPN}$ at an L:S ratio of $1.2-1.4 \mathrm{~mL} / \mathrm{g}$ had similar dispersion ability as PMMA. For L:S ratios less than $1 \mathrm{~mL} / \mathrm{g}$, the dispersion ability for both CPC and the CPN became low and the clump revealed irregular geometry. The fluidity and dispersion ability of cement determine its diffusion to the cancellous bone after its extrusion from the cannulated bone screw, eventually affecting the mechanical stability of the screw in the bone. L:S ratios greater than $0.8 \mathrm{~mL} / \mathrm{g}$ and less than $1.6 \mathrm{~mL} / \mathrm{g}$ are suggested for application of CPN in cannulated pedicle screws.

\section{Biomechanical properties of cement- augmented pedicle screws}

Axial pullout strength

Augmentation of cannulated and solid screws in Sawbones blocks by different bone cements was evaluated by axial pullout tests. The screw-cement complexes in the Sawbones blocks were observed by X-ray and PMMA, and CPC and the CPN exhibited similar morphology after augmentation. Comparing the radiographs of cannulated and solid screws, the bone cemented deployed through cannulated screws

Table 2 In vitro degradation results expressed by the weight loss of cements

\begin{tabular}{llllll}
\hline Soaking time & I week (\%) & 2 weeks (\%) & 3 weeks (\%) & 4 weeks (\%) & 5 weeks (\%) \\
\hline CPC & $6.00 \pm 0.55$ & $6.95 \pm 0.67$ & $7.77 \pm 0.56$ & $8.03 \pm 0.49$ & $8.72 \pm 0.37$ \\
CPN & $6.13 \pm 0.09$ & $7.12 \pm 0.52$ & $8.40 \pm 0.57$ & $9.58 \pm 0.60$ & $10.62 \pm 0.59$ \\
\hline
\end{tabular}

Abbreviations: CPC, calcium phosphate cement; CPN, calcium phosphate nanocomposite. 


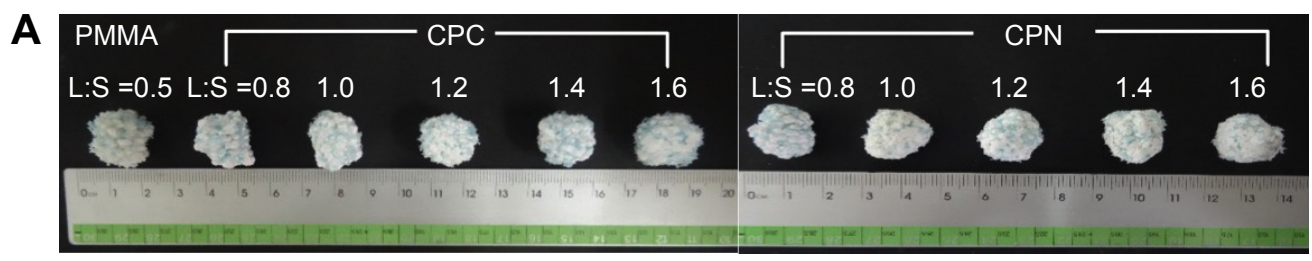

B

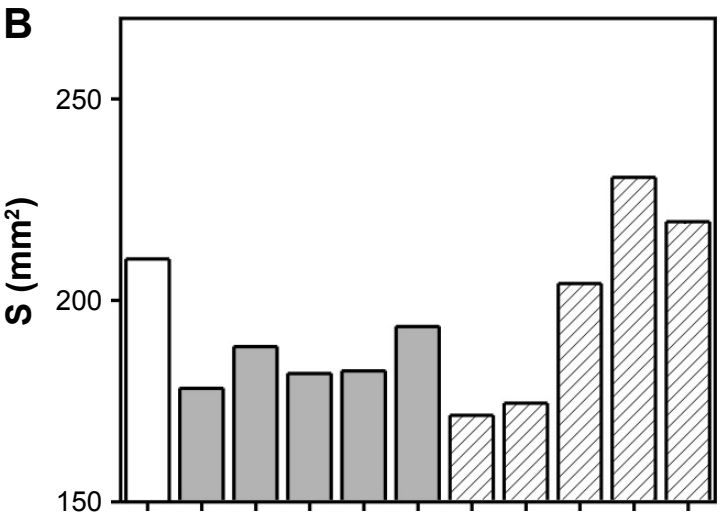

L:S

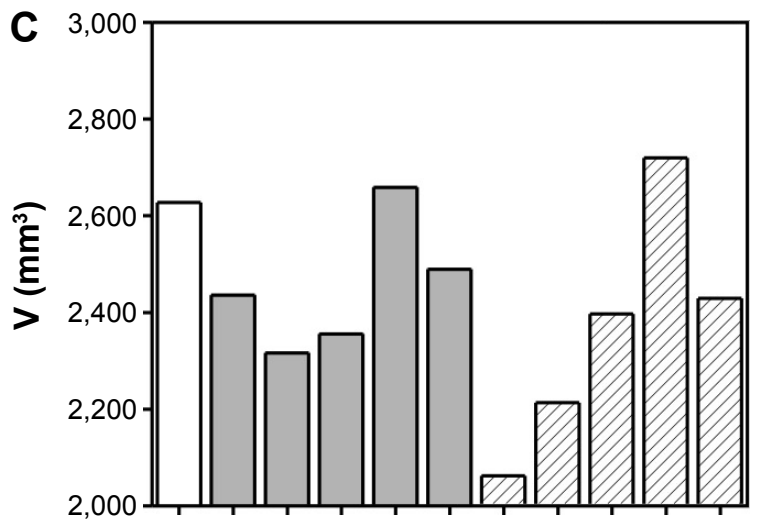

L:S

Figure 4 Fluidity and dispersion ability of bone cements tested in Sawbones blocks.

Notes: (A) Photographs of PMMA, CPC, and CPN lumps with different L:S ratios after dispersal and setting in Sawbones blocks. (B) Average projection areas and (C) estimated volumes of the bone-cement lumps.

Abbreviations: PMMA, polymethyl methacrylate; CPC, calcium phosphate cement; CPN, calcium phosphate nanocomposite; L:S, liquid:solid.

had more circular morphology, suggesting that the cement extruded from the cannula remained high fluidity, rendering its dispersion and interdigitation in the porous bone structure possible. It is worth mentioning that the CPN appeared to have similar morphology and dispersion ability as the PMMA (Figure 5), indicating the potential of the CPN for clinical application in the treatment of osteoporotic bones.
More importantly, the axial pullout strength of the CPN slightly outperformed PMMA in both cases of cannulated and solid screws, revealing adequate robustness and stability for application in the augmentation of pedicle screws. The representative pullout load-displacement curves shown in Figure 6 suggested that bone cement-augmented screws, either cannulated or solid, had dramatically increased pullout

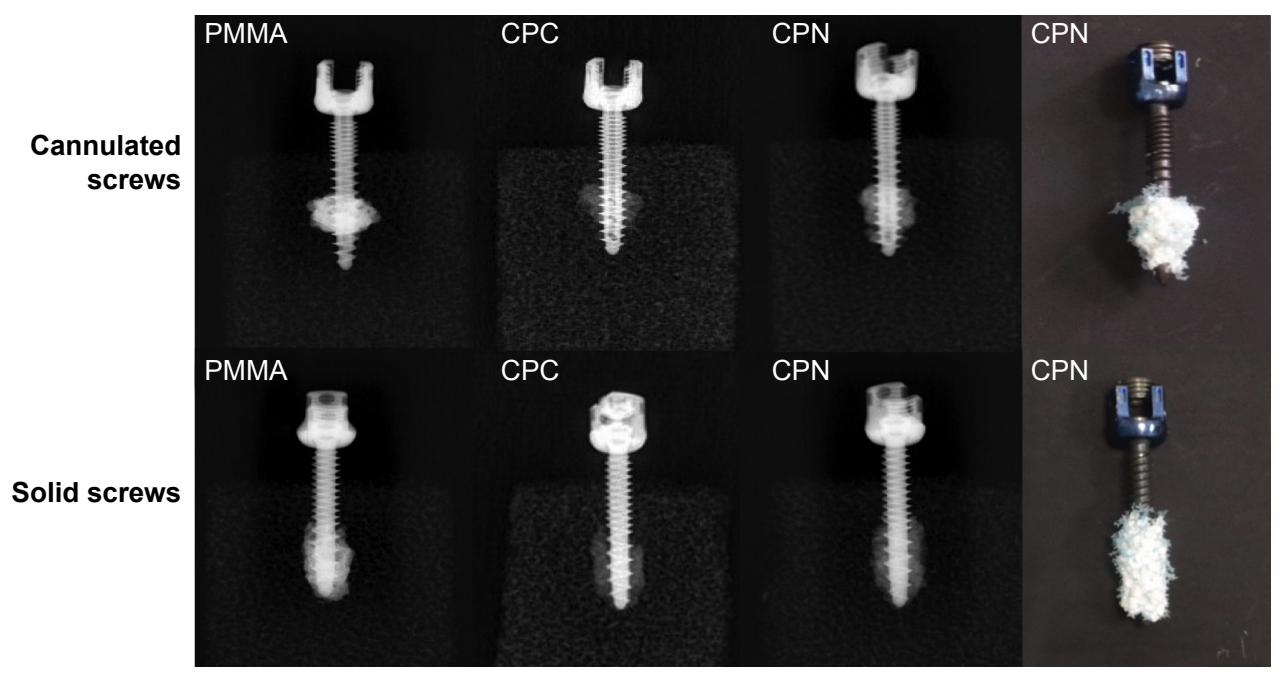

Figure $\mathbf{5}$ X-ray radiographs and photos of the augmentation of cannulated and solid screws in Sawbones blocks by different bone cements.

Note: Rightmost photos show the screw-cement complexes after axial pullout tests from Sawbones blocks.

Abbreviations: PMMA, polymethyl methacrylate; CPC, calcium phosphate cement; CPN, calcium phosphate nanocomposite. 

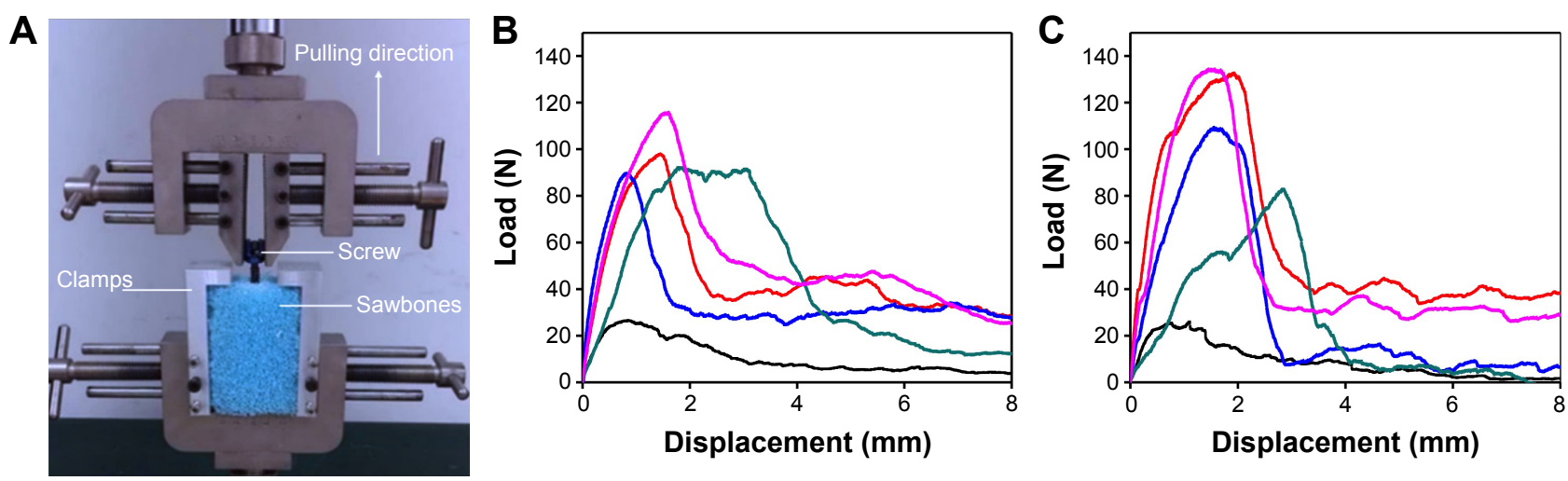

Blank OPMMA $\triangle \mathrm{CPC} \nabla \mathrm{CPC}-\mathrm{BS} \triangle \mathrm{CPN}$

Figure 6 Axial pullout tests on cement-augmented pedicle screws.

Notes: (A) Photo showing the setup of axial pullout test and representative load-displacement curves for (B) cannulated and (C) solid pedicle screws augmented without cement (Blank) and with PMMA, CPC, CPC-BS, and CPN.

Abbreviations: $\mathrm{PMMA}$, polymethyl methacrylate; $\mathrm{CPC}$, calcium phosphate cement; $\mathrm{BS}, \mathrm{BaSO}_{4}$; $\mathrm{CPN}$, calcium phosphate nanocomposite.

strength (i.e., maximum load during pulling) compared to unaugmented screws in the Sawbones blocks. Among the cement-augmented screws, the CPN showed the highest pullout strengths, which in turn indicated the highest antipullout ability of the CPN-augmented screws in osteoporotic bones.

Quantification results shown in Figure 7 clearly confirmed the observations in the pullout load-displacement curves. In the case of cannulated screws, the $\mathrm{CPN}$ reached the highest pullout strength of $\sim 120 \mathrm{~N}$, which was marginally higher than PMMA but significantly higher than CPC-BS or CPC $(P<0.05)$. This result also demonstrated the effect of adding nanoscale starch networks on improving the desired mechanical properties of CPC or CPC-BS for the augmentation of pedicle screws. In the case of solid screws, pullout strengths of the CPN, CPC, and PMMA were at a similar level (no significant difference), and pullout strengths were lower than in cannulated screws.

\section{Maximum torque}

Similarly, torsion tests were carried out to evaluate the antitorsion ability of bone cements when used for the augmentation of cannulated or solid pedicle screws. Typical torque-angle curves shown in Figure 8 suggest that bone cements significantly improved the antitorsion ability of both types of screws. The screws without cement-augmentation showed minimal torque values, with no ability to maintain mechanical stability during screw rotation. In contrast, PMMA resulted in the highest and the CPN the second-highest torque values for both cannulated and solid screws. Average results shown
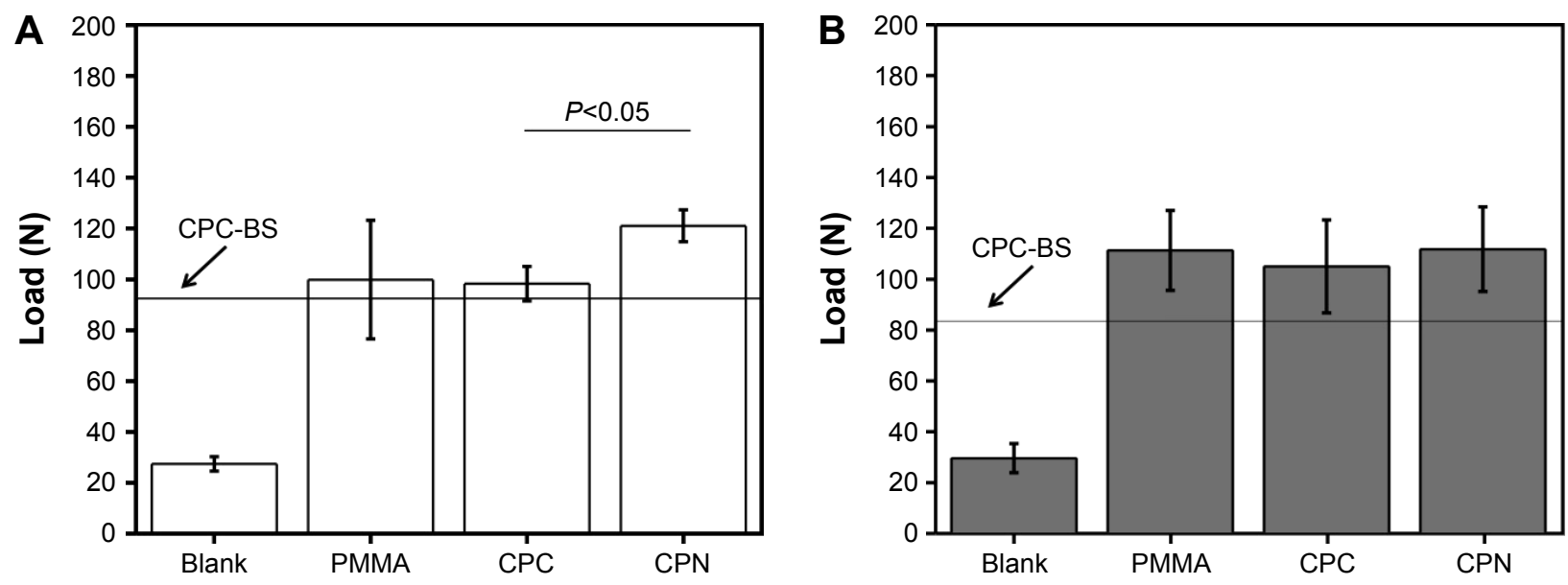

Figure 7 Axial pullout strengths of cement-augmented pedicle screws.

Notes: (A) Cannulated and (B) solid pedicle screws augmented without cement (Blank) and with PMMA, CPC, CPC-BS (indicated by the line) and CPN. The pullout strength was expressed by the maximum load reached during the pullout test. Data are mean \pm standard deviation $(n=5)$.

Abbreviations: PMMA, polymethyl methacrylate; CPC, calcium phosphate cement; $\mathrm{BS}, \mathrm{BaSO}_{4}$; $\mathrm{CPN}$, calcium phosphate nanocomposite. 

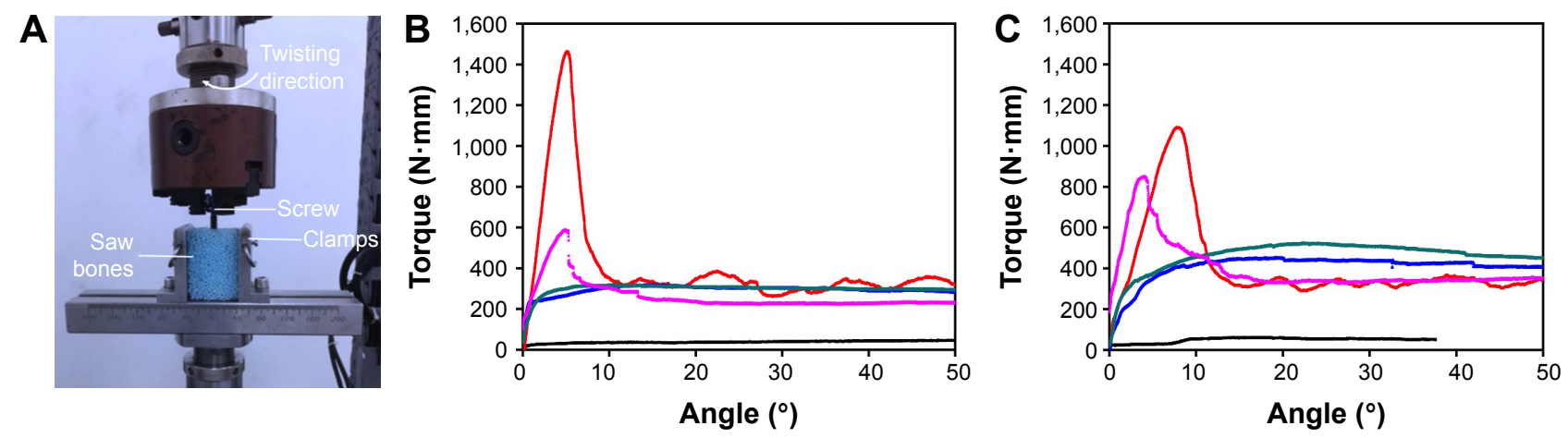

Blank - PMMA $\triangle$ CPC $\nabla$ CPC-BS 4 CPN

Figure 8 Torsion tests on cement-augmented pedicle screws.

Notes: (A) Photo of the setup of torsion tests. Representative torque-angle curves for (B) cannulated and (C) solid pedicle screws augmented without cement (Blank) and with PMMA, CPC, CPC-BS, and CPN.

Abbreviations: PMMA, polymethyl methacrylate; $\mathrm{CPC}$, calcium phosphate cement; $\mathrm{BS}, \mathrm{BaSO}_{4} ; \mathrm{CPN}$, calcium phosphate nanocomposite.

in Figure 9 are consistent with the observations in Figure 8, and the maximum torque of PMMA and CPN reached 1,400 $\mathrm{N} \cdot \mathrm{mm}$ and $600 \mathrm{~N} \cdot \mathrm{mm}$ for cannulated screws and $1,000 \mathrm{~N} \cdot \mathrm{mm}$ and $750 \mathrm{~N} \cdot \mathrm{mm}$ for solid screws, respectively. Also, the CPN showed much higher torque values than CPC or CPC-BS for both types of screws, again confirming the reinforcing effect of nanoscale starch networks on augmentation efficacy. Although the CPN did not outperform PMMA in antitorsion ability, its significant improvement compared to CPC or cementless screws may still guarantee its potential for the augmentation of pedicle screws in osteoporotic bones. In addition, the lower maximum torque of the CPN rendered the retrieval of screws from the cement possible, which is a current problem for PMMA, forming excessive bonding to screws.

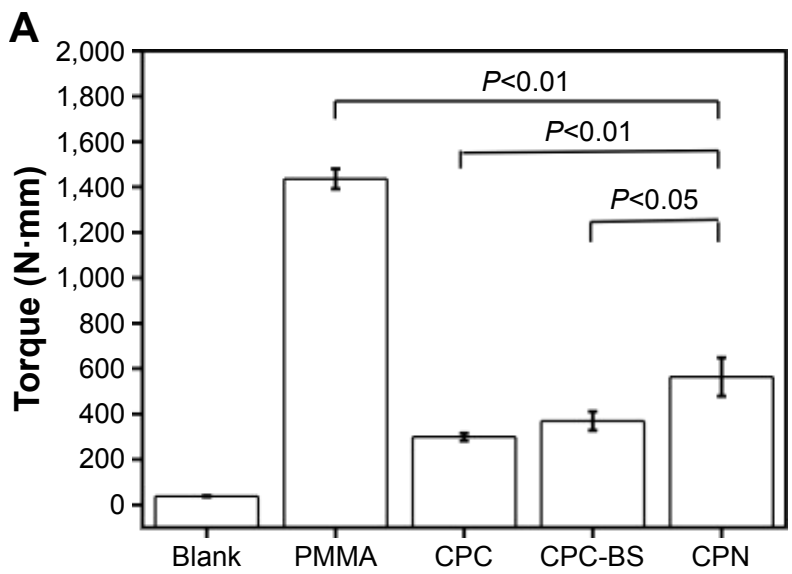

\section{In vivo osseointegration and degradation of CPN}

Given the well-known fact that PMMA does not degrade in vivo and cannot establish osseointegration with bone, animal-study results shown in Figure 10 demonstrated the superior in vivo osseointegration and degradability of the CPN in an 8-week rat femur-defect model. The H\&Estained CPN and bone tissue revealed that the boundary of an originally cylindrical CPN sample changed to one with irregular outlines and bone ingrowth was also found along such irregular boundaries (Figure 10A). Also, the bone surrounding the cement formed a tight contact with the cement, and a void also formed in the area close to the center of CPN (Figure 10B). These results evidently signaled the simultaneous degradation and bone ingrowth of $\mathrm{CPN}$ in vivo,

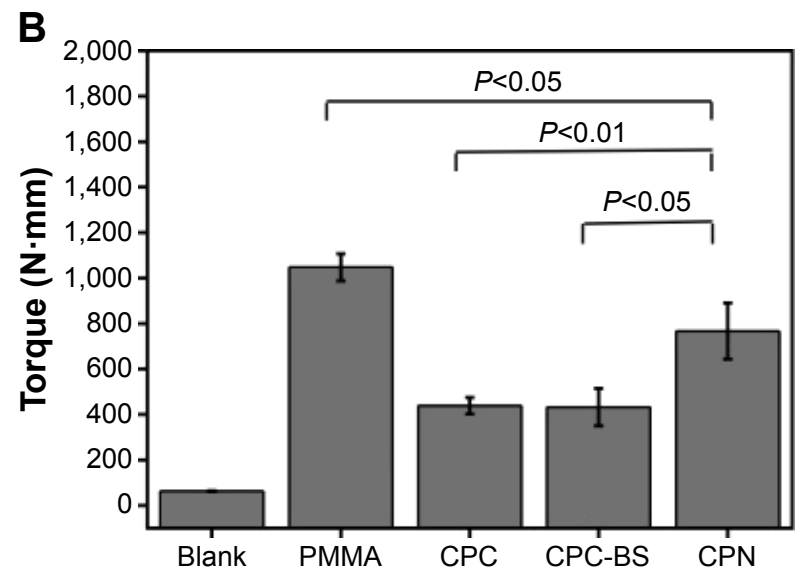

Figure 9 Maximum torque measured on cement augmented pedicle screws.

Notes: (A) Cannulated and (B) solid pedicle screws augmented without cement (Blank) and with PMMA, CPC, CPC-BS, and CPN. Data are mean \pm standard deviation $(n=5)$.

Abbreviations: PMMA, polymethyl methacrylate; CPC, calcium phosphate cement; $\mathrm{BS}, \mathrm{BaSO}_{4}$; $\mathrm{CPN}$, calcium phosphate nanocomposite. 

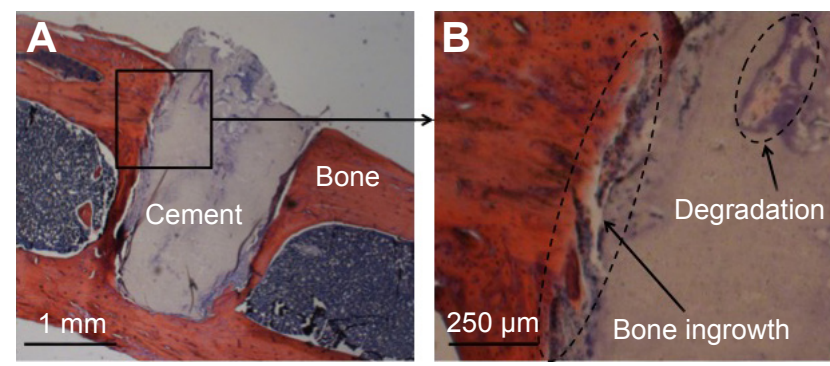

Figure 10 Histological analysis of the CPN implanted in rat-femur defect after 8 weeks, H\&E-stained.

Note: (A) The smooth outer surface of cement degraded, and (B) bone ingrowth was observed.

Abbreviations: CPN, calcium phosphate nanocomposite; H\&E, hematoxylin and eosin.

making the CPN a better augmenting material than PMMA, which can increase bone ingrowth into cannulated screws.

\section{Discussion}

The major advantage of PMMA-augmented cannulated pedicle screws is greatly increasing the mechanical stability of screws in osteoporotic vertebrae, and thus preventing pullout of screws during spine motion. ${ }^{4,5}$ The complications and drawbacks of PMMA have also gradually been unveiled during clinical practice in the past few years. ${ }^{1-3}$ Most drawbacks originate from the excessive mechanical strength or stiffness and nondegradability of PMMA, with its intrinsic material properties difficult to alter. This requires a new injectable material distinct from PMMA, but also be able to prevent pullout of screws from the vertebral body.

The results of the CPN demonstrated a number of promising properties for augmenting cannulated pedicle screws, providing a new approach to solve the current problems associated with PMMA. For application in augmenting cannulated pedicle screws, the nanocomposite possessed slightly better antipullout ability than PMMA and similar fluidity and dispersion ability in osteoporotic bone as PMMA. More importantly, the CPN was biodegradable and induced bone ingrowth after its degradation, which could be attributed to its composition of crystalline HA. Although further animal and clinical studies are needed, the results so far strongly support that CPN can be used for the augmentation of cannulated pedicle screws, and its long-term clinical outcomes are likely to be better than PMMA, due to its ability to promote bone growth into the cannulated screws. This bone ingrowth is expected to increase further the mechanical stability between vertebrae and pedicle screws and eventually contribute to the enhancement of spine stability, which will be investigated and validated in a future study.

Although the CPN was not as good as PMMA in antitorsion performance, it still outperformed other CP-based cements, with a significant increase in the maximum torque $(>60 \%)$. To our knowledge, there is no consensus or data reported on the torque values experienced by the cannulated particle screws in patients. Therefore, it is not reasonable to conclude that the CPN cannot provide sufficient antitorsion capacity at this stage. Also, the current literature appears to focus more on antipullout ability than antitorsion ability of screws, ${ }^{5-10}$ and thus the need for high antitorsion capacity is still unclear. This question needs more systematic investigation in the future.

It is worth mentioning that failure modes of $\mathrm{CPN}-$ and PMMA-screw complexes in the torsion tests were vastly different. Cannulated screws were detached from the CPN when the torque value reached maximum. In contrast, the screw still bonded with PMMA, and the PMMA-screw complex was detached from Sawbones block when the torque value reached maximum. The difference in failure mode indicates that PMMA formed a much stronger interface with screws than with bone, while the CPN was the opposite. Cement-screw and screw-bone interfaces are both important to the mechanical stability of pedicle-screw fixation, but their roles in short- and long-term clinical outcomes are still unclear. Further understanding of this issue would contribute to the improvement of both PMMA and the CPN for their application.

In addition, augmenting efficacy of bone cements on both cannulated and solid screws was studied here. Clinically, cannulated screw fixation is considered more efficient than other screw-fixation methods, including cement-augmented solid-screw fixation. ${ }^{21}$ The comparison between cannulated and solid screws in the present study also revealed that CPNaugmented cannulated screws had better antipullout ability than CPN-augmented solid screws. In terms of antitorsion ability, CPN-augmented solid screws were slightly better than $\mathrm{CPN}$-augmented cannulated screws, but the difference was not significant. These results are not contradictory to the current understanding in clinical practice that cementaugmented cannulated screw fixation performs better than other fixation strategies.

Finally, the results reported here also demonstrate the role of nanotechnology in the improvement of desirable properties of traditional CPC. The nanoscale network of gelatinized starch demonstrated positive effects on improving injecting, biomechanical, and biodegradation properties of CPC for the first time. These positive effects can be attributed to the reinforcing effects of elastic or ductile nanofibers added to the homogeneous matrix of ceramic. ${ }^{22,23}$ In regard to the toxicity and blood compatibility of the CPN, which are important 
for future clinical applications of a CPN, our previous and ongoing studies demonstrate that this $\mathrm{CPN}$ does not cause toxic or hemolytic effects in vitro. ${ }^{19}$ Therefore, to our knowledge, this $\mathrm{CPN}$ is the first nanocomposite to demonstrate promising potential for the augmentation of pedicle-screw fixation.

\section{Conclusion}

The present study demonstrated a new injectable, biodegradable, implantable nanocomposite for the augmentation of cannulated pedicle screws, exhibiting better antipullout ability and similar fluidity and dispersion ability compared to clinically used PMMA. The CPN outperformed conventional CPC in all mechanical properties and biodegradability pertinent to the application of cannulated pedicle-screw fixation. This CPN is the first nanocomposite of its kind to be specifically designed for the augmentation of pedicle-screw fixation, and the promising results reported here suggest its worth for further study and development for clinical use.

\section{Acknowledgments}

HLS would like to acknowledge the Scientific Research Foundation for the Returned Overseas Chinese Scholar, Ministry of Education of China $(2015,311)$. LY would like to acknowledge the National Natural Science Foundation of China (81622032, 51672184, and 51472279), the Priority Academic Program Development of Jiangsu High Education Institutions (PAPD), Jiangsu Innovation and Entrepreneurship Program, National Basic Research Program of China (973 Program, 2014CB748600), and the Jiangsu Six Peaks of Talents Program (2013-WSW-056).

\section{Disclosure}

The authors report no conflicts of interest in this work.

\section{References}

1. Galbusera F, Volkheimer D, Reitmaier S, Berger-Roscher N, Kienle A, Wilke HJ. Pedicle screw loosening: a clinically relevant complication? Eur Spine J. 2015;24(5):1005-1016.

2. Aghayev E, Zullig N, Diel P, Dietrich D, Benneker LM. Development and validation of a quantitative method to assess pedicle screw loosening in posterior spine instrumentation on plain radiographs. Eur Spine J. 2014;23(3):689-694.

3. Bredow J, Boese CK, Werner CM, et al. Predictive validity of preoperative CT scans and the risk of pedicle screw loosening in spinal surgery. Arch Orthop Trauma Surg. 2016;136(8):1063-1067.

4. Padányi C, Misik F, Papp Z, et al. [Treatment of osteoporotic vertebral compression fractures with PMMA-augmented pedicle screw fixation]. Ideggyogyaszati Szemle. 2015;68(1-2):52-58. Hungarian.

5. Henroteaux A, Racaru T, Martin D, Scholtes F, Dubuisson A, Kaschten B. Polymethylmethacrylate (PMMA) augmentation of the pedicle screws in patients with fractures of osteoporotic bone. About our experience. conference abstract, Société Belge de Neurochirurgie, Mar 29, 2014. Available from: http://hdl.handle.net/2268/165163. Accessed March 26, 2017.
6. Yi S, Rim DC, Park SW, Murovic JA, Lim J, Park J. Biomechanical comparisons of pull-out strengths after pedicle screw augmentation with hydroxyapatite, calcium phosphate or polymethylmethacrylate in the cadaveric spine. World Neurosurg. 2015;83(6):976-981.

7. Hashemi A, Bednar D, Ziada S. Pullout strength of pedicle screws augmented with particulate calcium phosphate: an experimental study. Spine J. 2009;9(5):404-410.

8. Cho W, Wu C, Erkan S, Kang MM, Mehbod AA, Transfeldt EE. The effect on the pullout strength by the timing of pedicle screw insertion after calcium phosphate cement injection. J Spinal Disord Tech. 2011;24(2):116-120.

9. Liu D, Lei W, Wu ZX, et al. Augmentation of pedicle screw stability with calcium sulfate cement in osteoporotic sheep: biomechanical and screw-bone interfacial evaluation. J Spinal Disord Tech. 2011; 24(24):235-241.

10. Güler UO, Derincek A, Hersekli MA, Ozalay M, Cinar BM, Acaroğlu E. Restoration of pull-out strength of the failed pedicle screw: biomechanical comparison of calcium sulfate vs polymethylmethacrylate augmentation. Acta Orthop Traumatol Turc. 2014;48(2):202-206.

11. Pare PE, Chappuis JL, Rampersaud R, et al. Biomechanical evaluation of a novel fenestrated pedicle screw augmented with bone cement in osteoporotic spines. Spine (Phila Pa 1976). 2011;36(36):1210-1214.

12. Amendola L, Gasbarrini A, Fosco M, et al. Fenestrated pedicle screws for cement-augmented purchase in patients with bone softening: a review of 21 cases. J Orthop Traumatol. 2011;12(4):193-199.

13. Blattert TR, Glasmacher S, Riesner HJ, Josten C. Revision characteristics of cement-augmented, cannulated-fenestrated pedicle screws in the osteoporotic vertebral body: a biomechanical in vitro investigation technical note. J Neurosurg Spine. 2009;11(11):23-27.

14. Colman M, Pond J, Bachus K, Lawrence BD, Spiker WR, Brodke DS. Fenestrated screws augmented with PMMA increase the pull-out strength of sacral pedicle screws. Clin Spine Surg. Epub 2016 Jul 14.

15. Alvarez L, Gomez S, Vlad MD, Pinera AR, Tome-Bermejo F, Fernández E. Analysis of PMMA distribution around cannulated and fenestrated cement-augmented pedicle screws: clinical study. Spine J. 2015;15(10):S189-S190.

16. Piñera AR, Duran C, Lopez B, Saez I, Correia E, Alvarez L. Instrumented lumbar arthrodesis in elderly patients: prospective study using cannulated cemented pedicle screw instrumentation. Eur Spine J. 2011; 20(3):408-414.

17. Waits C, Burton D, Mciff T. Cement augmentation of pedicle screw fixation using novel cannulated cement insertion device. Spine (Phila Pa 1976). 2009;34(14):E478-E483.

18. Gao C, Liu H, Yang H, Yang L. Fabrication and characterization of injectable calcium phosphate-based cements for kyphoplasty. Mater Technol. 2015;30 (Suppl 5):256-263.

19. Liu H, Guan Y, Wei D, Gao C, Yang H, Yang L. Reinforcement of injectable calcium phosphate cement by gelatinized starches. J Biomed Mater Res B Appl Biomater. 2016;104(3):615-625.

20. Chen LH, Tai CL, Lee DM, et al. Pullout strength of pedicle screws with cement augmentation in severe osteoporosis: a comparative study between cannulated screws with cement injection and solid screws with cement pre-filling. BMC Musculoskelet Disord. 2011;12:33.

21. Dai F, Liu Y, Zhang F, et al. Surgical treatment of the osteoporotic spine with bone cement-injectable cannulated pedicle screw fixation: technical description and preliminary application in 43 patients. Clinics (Sao Paulo). 2015;70(2):114-119.

22. Yang L. Nanotechnology-Enhanced Orthopedic Materials: Fabrications, Applications and Future Trends. 1st ed. Cambridge, UK: Woodhead Publishing; 2015.

23. Yang L, Zhang LJ, Webster TJ. Nanobiomaterials: state of the art and future trends. Adv Eng Mater. 2011;13(6):B197-B217. 


\section{Publish your work in this journal}

The International Journal of Nanomedicine is an international, peerreviewed journal focusing on the application of nanotechnology in diagnostics, therapeutics, and drug delivery systems throughout the biomedical field. This journal is indexed on PubMed Central,

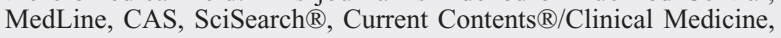

Journal Citation Reports/Science Edition, EMBase, Scopus and the Elsevier Bibliographic databases. The manuscript management system is completely online and includes a very quick and fair peer-review system, which is all easy to use. Visit http://www.dovepress.com/ testimonials.php to read real quotes from published authors.

Submit your manuscript here: http://www.dovepress.com/international-journal-of-nanomedicine-journal 